\title{
TRES EN UNO, ¿O SON MÁS? HISTORIA DE EPIDENDRUM DICHOTOMUM LINDL., NON PRESL
}

\author{
Gustavo A. Romero-GonzÁlez' \& Germán Carnevali Fernández-ConchA² \\ ${ }^{1}$ Orchid Herbarium of Oakes Ames, Harvard University Herbaria, 22 Divinity Avenue \\ Cambridge, Massachussets 02138, U.S.A. romero@oeb.harvard.edu \\ ${ }^{2}$ Herbarium CICY. Unidad de Recursos Naturales, Centro de Investigación Científica de Yucatán A. C. (CICY) \\ Ap. Postal 87, Mérida 97310, Yucatán, México. carneval@cicy.mx
}

El siguiente ensayo dio inicio cuando uno de los autores (G.C.) localizó un ejemplar no identificado de Epidendrum de Guyana en el herbario del Smithsonian (US). El ejemplar fue trasladado en préstamo a AMES y luego de un breve análisis llegamos a la conclusión de que era muy parecido a una especie que John Lindley había descrito en 1838 como Epidendrum dichotomum. Luego de una revisión de literatura y de material de herbario disponible de esta especie, se hizo evidente que estábamos tratando no con una sola entidad, sino con un "basurero", es decir, una especie múltiple (en inglés, "a catch-all name"), aquélla que por error o conveniencia incluye varias otras especies relacionadas, algunas de las cuales con frecuencia nunca han sido descritas formalmente. En este caso en particular, el nombre que ultimadamente se le daría al Epidendrum dichotomum de Lindley, nomenclatorial y taxonómicamente incluiría al menos tres especies obviamente diferentes.

Lindley basó su Epidendrum dichotomum en dos ejemplares: uno de Brasil (G. Gardner 631) y otro de Guyana (cultivado en el establecimiento de Conrad Loddiges \& Hijos). Sin embargo, ya este nombre había sido utilizado por Presl en 1827 para una especie de Perú. Al enterarse de la publicación de Pres1, Lindley propone en 1853 el nombre Epidendrum corymbosum para su especie. En esta descripción, que no es igual a la publicada en 1838 (Fig. 1A-B), Lindley citó dos ejemplares adicionales: uno de Brasil, sin procedencia precisa (Miers 3484) y otro de los Andes de Venezuela (Linden 647). Desafortunadamente para Lindley, el nombre nuevo que propuso ya también había sido utilizado por Ruiz \& Pavón en 1798 para otra especie de Perú. Un poco más de cien años más tarde, Pabst (en Angely 1956) propone Epidendrum ecostatum como un nombre nuevo para E. corymbosum Lindl., non Ruiz \& Pavón, pero sin seleccionar un tipo. Esta propuesta aparentemente resolvía los problemas nomenclatoriales de Epidendrum dichotomum Lindl., non Presl, y de Epidendrum corymbosum Lindl., non Ruiz \& Pavón. Sin embargo, un examen detallado de los cuatro ejemplares que Lindley citó en sus descripciones de 1838 y 1853 indica que éstos representan las siguientes especies (las especies inéditas serán tratadas en un manuscrito aparte; Romero \& Carnevali, en preparación):

Epidendrum praetervisum Rchb.f., Linnaea 41: 82. 1876. TIPO: VenEZuELA. Mérida: "bosques en los alrededores de Mérida", 1842, J. J. Linden 647 (Holotipo: Reichenbach Herbarium Nr. 4359; Isotipo: K-Lindl.). Fig. 1C.

ILUSTRACIÓN: G. C. K. Dunsterville 703 en Romero y Carnevali (2000: 278).

Esta es la especie o, más precisamente, el tipo más fácil de separar de Epidendrum dichotomum Lindl., non Presl, y de E. corymbosum Lindl., non Ruiz \& Pavón. Las plantas generalmente se encuentran a mayores alturas y son bastante diferentes, floralmente, a lo que Lindley originalmente intentó incluir bajo esos dos nombres. Se conoce de Venezuela y probablemente se halla en Colombia también.

Epidendrum ecostatum Pabst [en J. Angely], Orchidaceae Paranaensis. Flora do Parana 6: 11. 1956.

Basiónimo: Epidendrum corymbosum Lindl., non Ruiz \& Pavón, Fol. Orchid. Epidendrum 61. 1853. TIPO: BRASIL. Sin localidad precisa, J. Miers 3484 
(Lectotipo, propuesto, de facto, por Ackerman, 1995, K-Lindl.; fragmento, AMES).

Sinónimo: Epidendrum corymbosum Lindl., non Ruiz \& Pavón var. latifolium Cogn., Fl. Bras. (Martius) 3, 5: 144. 1888. TIPO: BRASIL. Sin localidad precisa ["In Brasilia austro-orientale" fide Cogniaux], E. F. M. Glaziou 11623, pro parte (Holotipo: BR; fotocopia, AMES).

ILUSTRACIÓN: Cogniaux, Fl. Bras. (Martius) 3, 5: t. 44, Fig. 2. 1888; Pabst \& Dungs (1975: 304 , dibujo 726).

La descripción publicada por Lindley en $1853 \sin$ duda alguna se refiere a la planta colectada por Miers, que está montada en una cartulina aparte en su herbario depositado en Kew; en ésta Lindley dibujó una flor de esta muestra. El dibujo de Pabst \& Dungs (1975: 304, dibujo 726) también muestra bastante bien la morfología floral de Epidendrum ecostatum. En esta especie el labelo es ligera a conspicuamente trilobulado y el ápice del lóbulo central es truncadoredondeado y tiene un pequeño apículo en el ápice de la superficie abaxial.

Foldats (1975: 243-244) citó Epidendrum corymbosum Lindl. de Venezuela siguiendo a Schlechter (1919: 68), quien registró esta especie de la Cordillera de la Costa Central ("Federal-District; Miranda"), seguramente refiriéndose a otra especie de Epidendrum.

Epidendrum jamaicense Lindl., Fol. Orch. Epidendrum 82. 1853. TIPO: JamaICA. "On trees in the woods of Dunrobin Castle", W. Purdie s.n. (Holotipo: K-Hook.; dibujo, K-Lindl.).

Sinónimo: Epidendrum corymbosum auct., non Lindl., non Ruiz \& Pavón; Stehlé, Fl. Descript. Ant. Franc. 1: 148-149. 1939; Cogniaux, Symb. Antillan. 6: 525.1810 .

ILUSTRACIÓN: Stehlé, Fl. Descript. Ant. Franc. 1: 150-151. 1939.

Esta especie de las Antillas, que antes era identificada como "Epidendrum corymbosum Lindl." (Cogniaux 1810, Stehlé 1839), ahora es referida a Epidendrum jamaicense (Garay \& Sweet 1974: 141, Ackerman 1995: 68-69).
Epidendrum sp. 1. Espécimen de referencia: GUYANA ["Demerara"]. Sin localidad precisa, [R. H. Schomburgk] ex Hort. Loddiges (K-Lindl., montado en la misma cartulina en la que se encuentran Gardner 631 y Linden 647, en el herbario de Lindley).

Sinónimo: Epidendrum ecostatum auct., non Pabst, E. A. Christenson, Mem. New York Bot. Gard. 76, 1: 305. 1997.

Esta es la especie a la que pertenece el ejemplar de US, en la que las plantas son mucho más pequeñas, con hojas angostas, y el labelo conspicuamente agudo y sin apículo en el ápice. Irónicamente, el dibujo de Lindley que se encuentra en la misma cartulina donde está el espécimen de referencia corresponde a esta especie, que no concuerda con ninguna de las dos descripciones publicadas por Lindley.

Epidendrum sp. 2. Espécimen de referencia: BRASIL. Río de Janeiro: Sierra de los Órganos, G. Gardner 631 (K-Lindl. [ver especie anterior], BM).

La descripción de Epidendrum dichotomum Lind1., non Presl, concuerda bastante con este espécimen, especialmente, como lo ha notado el Dr. Garay (comun. pers., 2003), por la presencia de un nectario que se prolonga hasta el ovario pedicelado ("ovario cuniculato"), que no se discierne en la única flor del lectotipo de Epidendrum ecostatum que ha examinado uno de nosotros (G.A.R.). En el espécimen de referencia de Epidendrum sp. 2 el labelo, como en E. ecostatum, es ligeramente trilobulado y el ápice del lóbulo central es truncadoredondeado y tiene un pequeño apículo en el ápice de la superficie abaxial. Si se detecta un nectario en el ovario pedicelado en otras flores del tipo de Epidendrum ecostatum, tal vez no sea necesario describir esta especie.

El caso que hemos relatado es un patrón que se repite en muchos grupos de orquídeas. Los autores ya han trabajado en varias de estas especies múltiples (Epidendrum nocturnum Jacq.: Carnevali \& Romero 1996; Cyrtopodium punctatum (L.) Lindl.: Romero \& Carnevali 1999; Galeandra beyrichii Rchb.f.: Romero \& Brown 2000). La existencia de estos casos confirma que están equivocados aquéllos que creen que taxonómicamente todo está resuelto en las 
A 146. EPIDẼNDRŨM dichŏtómüm.

E. dichotomum ; fruticosum, caule fruticoso decumbente filiformi dichotomo, foliis angustè lanceolatis acutissimis corymbo terminali longioribus, sepalis lineari-lanceolatis, petalis conformibus angustioribus, labello cuniculato cordato obtusiusculo basi bicalloso.

A green-flowered species obtained from Demerara by Messrs. Loddiges, and found in the Organ Mountains of Brazil by Mr. Gardner, (No. 631.) It has no beauty, but is remarkable for its hard, wiry, forking stem.

B * 190. E. corymbosum.

E. caule ramosissimo, foliis lanceolatis acuminatis racemo corymboso longioribus, pedunculo basi 1-2-bracteato, bracteolis setaceis pedicellis brevioribus, floribus membranaceis, sepalo supremo angusto lateralibus latioribus semiovalibus acutis petalis linearibus basi tenuissimis uninerviis, labello subrotundo cordato obtuso, callis 2 lineâque brevi elevatâ interjectâ.

E. dichotomum. Lindley, in Bot. Reg. 1838, misc. no. 146 ; not of Presl.

Wild in BraziL-Miers, no. 3484; Organ Mountains, on an old tree-Gardner, 631; Demerara-Loddiges ; Venezuela, on trees in the forests of Merida at 5500 feet-Linden, 647, (v. s. sp. et v.c.)

A small branching species with the appearance of $E$. anisatum; but the leaves are thinner, the bracts setaceous, and the lip quite membranous without lobes. Flowers green.

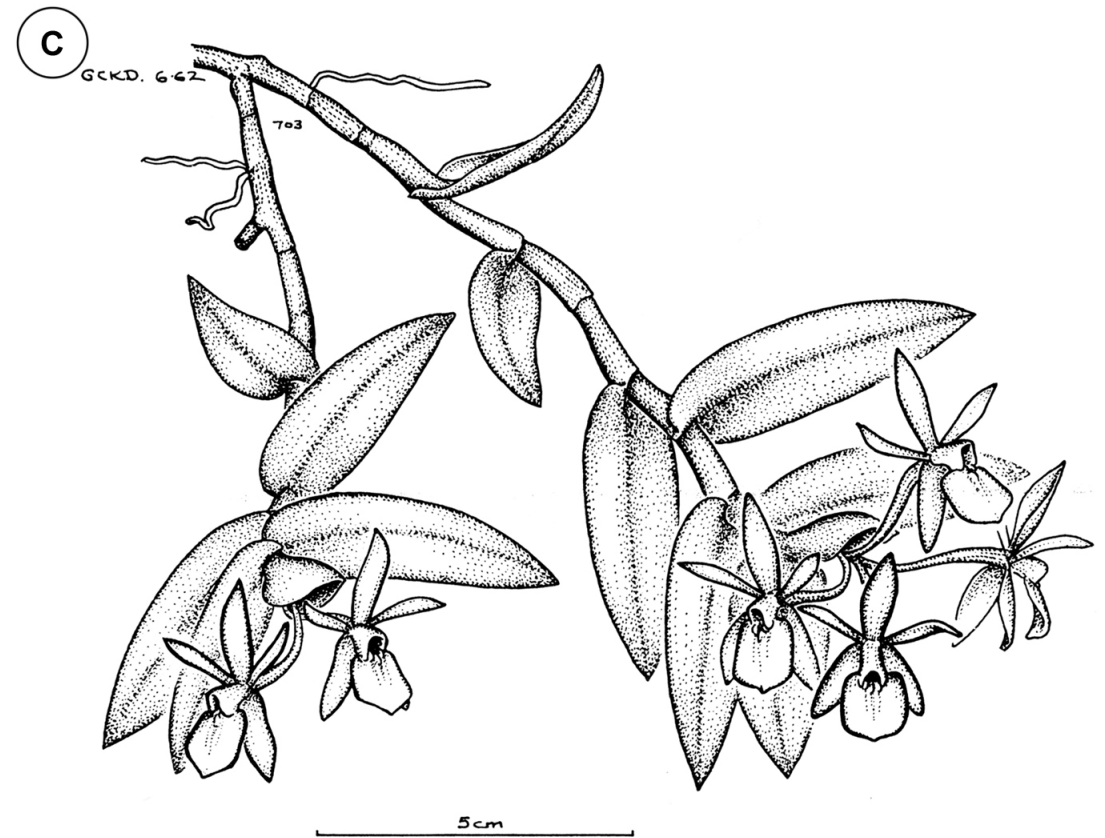

Figura 1. Epidendrum dichotomum Lindl., non Presl. A. Descripción del primer nombre de Lindley en Edwards's Bot. Reg. 24: 79 (1838). B. Descripción del segundo nombre de Lindley en Folia Orchid. Epidendrum 61 (1853). C. Epidendrum praetervisum Lindl. (dibujo de G. C. K. Dunsterville, basado en su colección número 703, AMES). 
orquídeas. Es más, nos atreveríamos a especular que, luego de llegar a esa ilusoria meta (i.e., donde todo está resuelto), Orchidaceae sin duda sería la familia más diversa de las plantas vasculares.

\section{Agradecimientos}

Los autores agradecen a los herbarios mencionados por su ayuda desinteresada, a K.N. Gandhi por su experticia en nomenclatura, y a L.A. Garay por proporcionar material invaluable para entender este grupo de especies.

\section{LITERATURA CITADA}

Ackerman, J. D.1995. An Orchid Flora of Puerto Rico and the Virgin Islands. Mem. New York Bot. Gard. 73: 1-203.

Carnevali, G. \& G.A. Romero. 1996. Orchidaceae Dunstervilleorum VII: the Epidendrum nocturnum alliance in the Venezuelan Guayana. Lindleyana 11: 239-249.

Cogniaux, A. 1910. Epidendrum corymbosum. Symb. Antillan. 6: 525-526.

Foldats, E. 1970. Orchidaceae. In T. Lasser (ed.), Flora de Venezuela 25, 3: 1-522. Instituto Botánico, Caracas.

Garay, L.A. \& H.R. Sweet. 1974. Orchidaceae. Volume 1. In R.A. Howard (ed)., Flora of the Lesser Antilles. Arnold Arboretum, Harvard University, Jamaica Plains, Massachusetts.
Lindley, J. 1838. Epidendrum dichotomum. Edwards's Bot. Reg. 24: 79 .

Lindley, J. 1853. Epidendrum corymbosum. Folia Orch. Epidendrum 61.

Pabst, G.F.J. 1956. Epidendrum ecostatum. p. 11 in J. Angely (ed.), Orchidaceae Paranaensis. Flora do Parana 6: 11.

Pabst, G.FJ. \& F. Dungs. 1975. Orchidaceae Brasilienses I. Brücke-Verlag Kurt Schmersow, Hildesheim.

Schlechter, R. 1919. Die Orchideenfloren der Südamerikanischen Kordillerenstaaten I. Venezuela. Repert. Spec. Nov. Regni Veg. Beih. 6: 1-100.

Stehlé, H. 1939. F1. Descript. Ant. Franc. 1. Les Orchidales. Imprimerie Oficielle de la Martinique, Fortde-France.

Romero-González, G.A. \& G. Carnevali FernándezConcha. 1999. Notes on the species of Cyrtopodium (Cyrtopodinae, Orchidaceae) from Florida, the Greater Antilles, Mexico, Central and Northern South America. Harvard Pap. Bot. 4: 327-341.

Romero-González, G.A. \& G. Carnevali FernándezConcha. 2000. Orquídeas de Venezuela [una Guía de Campo Ilustrada]. Armitano Editores, Caracas.

Romero-González, G.A. \& P. M. Brown. 2000. Galeandra bicarinata, a new species from Florida and the Greater Antilles. North Amer. Native Orch. J. 6: 7787.

Gustavo A. Romero-González es Curador del Orchid Herbarium de Oakes Ames y Editor de Harvard Papers in Botany. Actualmente está realizando trabajos monográficos y florísticos sobre las Orchidaceae de la región septentrional de Sudamérica, así como investigación sobre las bases biológicas para el manejo a largo plazo de los productos forestales no maderables en el Neotrópico (incluyendo las orquídeas, por supuesto!).

Germán Carnevali obtuvo su Licenciatura en Biología en la Universidad Central de Venezuela; Maestría y Doctorado en la Universidad de Missouri-St. Louis, asociado con el Missouri Botanical Garden. Sus intereses son sistemática y filogenia de varios grupos de Orchidaceae Neotropicales, principalmente los géneros Myrmecophila, Schomburgkia, Encyclia, Lophiaris, Cohniella y la subtribu Maxillariinae en general. Simultáneamente, tiene intereses en la florística de las Orchidaceae de la Península de Yucatán, América Central, las Guianas, la Amazonía y Venezuela. 